\title{
Autosomal recessive nail dysplasia
}

INSERM

\section{Source}

INSERM. (1999). Orphanet: an online rare disease and orphan drug data base. Autosomal recessive nail dysplasia. ORPHA:280654

Autosomal recessive nail dysplasia is a rare, isolated nail anomaly characterized by clawshaped, thick, hyperplastic, hard and hyperpigmented nails, subungual hyperkeratosis, onycholysis and slow nail growth. Variable degree of disease severity has been reported. 